\title{
Algebra of Classical and Quantum Binary Measurements
}

\author{
Carl A. Brannen \\ Retired, Henderson, NV, USA \\ Email:carl.brannen@wsu.com
}

How to cite this paper: Brannen, C.A. (2018) Algebra of Classical and Quantum Binary Measurements. Journal of Modern Physics, 9, 628-650.

https://doi.org/10.4236/jmp.2018.94044

Received: January 31, 2018

Accepted: March 24, 2018

Published: March 27, 2018

Copyright $\odot 2018$ by author and Scientific Research Publishing Inc. This work is licensed under the Creative Commons Attribution International License (CC BY 4.0).

http://creativecommons.org/licenses/by/4.0/

\section{cc) (i) Open Access}

\begin{abstract}
The simplest measurements in physics are binary; that is, they have only two possible results. An example is a beam splitter. One can take the output of a beam splitter and use it as the input of another beam splitter. The compound measurement is described by the product of the Hermitian matrices that describe the beam splitters. In the classical case, the Hermitian matrices commute (are diagonal) and the measurements can be taken in any order. The general quantum situation was described by Julian Schwinger with what is now known as "Schwinger's Measurement Algebra". We simplify his results by restriction to binary measurements and extend it to include classical as well as imperfect and thermal beam splitters. We use elementary methods to introduce advanced subjects such as geometric phase, Berry-Pancharatnam phase, superselection sectors, symmetries and applications to the identities of the Standard Model fermions.
\end{abstract}

\section{Keywords}

Quantum Mechanics, Schwinger Measurement Algebra, Quantum Thermodynamics

\section{Introduction}

In 1955, Julian Schwinger began work on the foundations of quantum field theory while employed at Harvard. The result was what is now known as "Schwinger's Measurement Algebra". The algebra was described in four of his 1959-1960 papers: [1] [2] [3] [4]. Schwinger used his algebra to teach introductory quantum mechanics. He joined the faculty at the University of California, Los Angeles in 1972 and his lecture notes from there resulted in two textbooks [5] [6] that many of his students then used when they taught introductory quantum mechanics. These textbooks cover the usual subjects in a standard 
quantum mechanics class. Where they are distinct is in their introduction to the subject, the algebra that this paper expands.

Schwinger's first paper "The Algebra of Microscopic Measurements" [1] describes his measurement algebra. His measurements can be thought of as beam splitters where one is concerned with only one of the exits. The algebra relates to how one models a complex beam splitter that consists of a series of beam splitters connected together by arranging for the output of one beam splitter to be used as the input of the next. The reader of Schwinger's paper will note that while his notation is different, the properties of the elements of his algebra are similar to those of mixed density matrices. We will use density matrix notation in this paper. We expand Schwinger's results to include the classical situation as well as imperfect and thermal beam splitters. To simplify the discussion, we will mostly consider binary measurements.

There are two reasons for reading this paper. The first is that Schwinger's beam splitter model provides the most direct method of passing from the classical to the quantum domain. Since Schwinger's method is completely general for quantum mechanics and quantum field theory, this provides an immediate connection between the classical and quantum situations and may provide an improved understanding of the foundations of quantum mechanics for students. In addition, binary measurements are arguably the easiest introduction to quantum physics and we introduce subjects that usually require much more preparation such as geometric phase, Berry-Pancharatnam phase and quantum statistics. The second reason is that some problems in quantum mechanics are far easier to understand in one formulation than in the others [7]. Schwinger's formulation is particularly useful in putting the symmetries of quantum mechanics on an algebraic basis. We demonstrate this by introducing a Schwinger algebra model of the elementary fermions.

\subsection{Beam Splitters}

The physical apparatus we're considering is a beam splitter. The beam splitter has a single entrance and two exits. Entering particles must take one of the two exits. Suppose that the particles are all identical of type " 1 " and that they do not influence one another and that they arrive at a beam splitter with a rate $\left|A_{1}\right|^{2}$. We use $\left|A_{1}\right|^{2}$ here (instead of $A_{1}$ ) for our rates in order to match the notation in the physics literature for beam splitters described with quantum mechanics. Our $A_{1}$ is the square root of a rate, so its units are $\sqrt{\text { particles/second }}$. In general, $A_{1}$ may be complex.

Suppose that a particle has a probability $p_{1}$ of exiting the upper exit so that the probability of exiting the lower exit is $1-p_{1}$. Then the rate of particles in the upper exit beam is $\left|B_{1}\right|^{2}=p_{1}\left|A_{1}\right|^{2}$ and the rate at the other exit is $\left(1-p_{1}\right)\left|A_{1}\right|^{2}=\left|B_{1}\right|^{2}-\left|A_{1}\right|^{2}$. See Figure 1 .

If we know the input rate and the upper exit output rate, then we can obtain the lower exit output rate by subtraction. Accordingly, for the remainder of this 


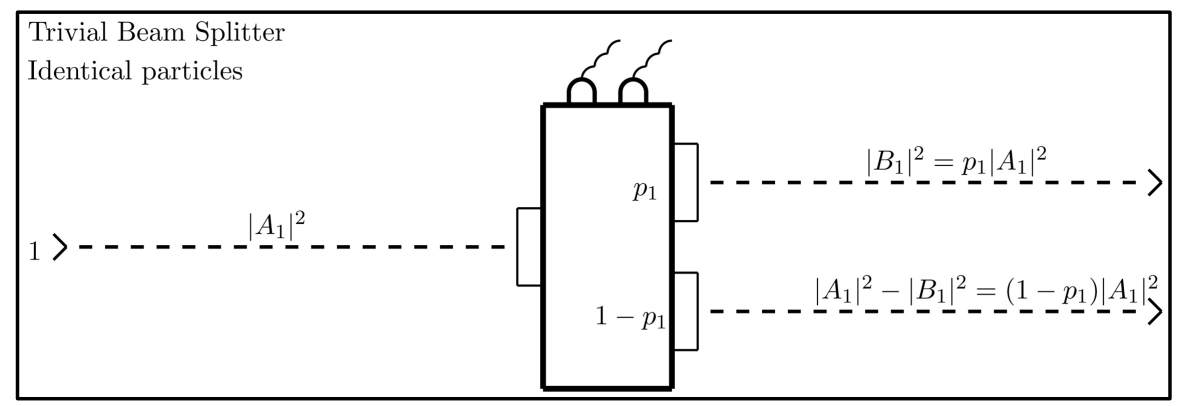

Figure 1. Trivial beam splitter splits a beam of type 1 particles arriving at a rate $\left|A_{1}\right|^{2}$. Particles take the upper exit with probability $p_{1}$ so the output rate for the upper exit is $\left|B_{1}\right|^{2}=p_{1}\left|A_{1}\right|^{2}$ and the output rate for the lower exit is $\left|A_{1}\right|^{2}-\left|B_{1}\right|^{2}=\left(1-p_{1}\right)\left|A_{1}\right|^{2}$. We've written the equations in terms of amplitudes $A_{j}$ rather than rates $\left|A_{j}\right|^{2}$ in order to match the standard quantum beam splitter notation.

paper, we will ignore the lower exit and concentrate only on the upper exit. For clarity, our drawings will continue to include the lower exit but we will not write formulas for it.

\subsection{Outline}

Schwinger noted that what makes quantum measurements different from classical is that a quantum measurement of one property (say spin in the $+Z$ direction) can disturb the system so that the results of a previous quantum measurement (of spin in another direction), no longer applies. Schwinger considered compound beam splitters obtained by putting the output of a first beam splitter into the input of a second beam splitter. He showed that these experiments can be represented by Hermitian matrices and that a compound beam splitter is represented by the product of its constituent beam splitter matrices.

In general, Hermitian matrices do not commute. For the Schwinger measurement algebra this means that changing the order of measurements can have a physical effect. If in addition to being Hermitian the matrices are also diagonal, then they will commute and the measurement order does not matter. In Section 2, we consider classical beam splitter experiments with imperfections or at temperature. We show that these experiments can be modeled with diagonal Hermitian matrices, that is, with real diagonal matrices.

Section 3 continues the analysis to the quantum case by allowing for nonzero off diagonal entries in the matrices. For simplicity, we specialize to spin-1/2 Stern-Gerlach experiments. We find that the quantum situation is a natural extension of the classical situation. We show why quantum mechanics uses complex numbers and amplitudes instead of probabilities, and we introduce the ideas of geometric phase, Berry-Pancharatnam phase, superselection sectors, quantum symmetries and quantum statistics.

As a unique formulation of quantum mechanics, Schwinger's measurement 
algebra can be expected to provide unique applications to Nature. Our analysis of superselection sectors concludes that their algebras are block diagonal in form. This suggests that we reverse the process. We can start with an algebra and from it derive the particle content. We include Section 4 as a speculation on the nature of the Standard Model fermions and dark matter. The paper concludes with a conclusion and acknowledgements.

\section{Classical Beam Splitters}

The two outputs of the beam splitter of Figure 1 have intensities $\left|B_{1}\right|^{2}$ and $\left|A_{1}\right|^{2}-\left|B_{1}\right|^{2}$. These intensities add up to $\left|A_{1}\right|^{2}$, the intensity of the original beam. If we combine the two exits back together we obtain an exit beam with intensity $\left|A_{1}\right|^{2}$ and expect that this beam will be indistinguishable from the original beam.

The beam splitter of Figure 1 is described with only a single number, the probability of a particle leaving the top exit, $p_{1}$. Suppose we have another beam splitter, this one with the corresponding probability $q_{1}$. Assuming independence, a particle will make it through both beam splitter's top exits with probability $q_{1} p_{1}$. See Figure 2. This process can be continued with any number of consecutive beam splitters. If we have $n$ beam splitters with probability $p_{1}$, the probability of a particle making it through all of them is $\left(p_{1}\right)^{n}$.

\subsection{Classical Thermal Beam Splitters}

To introduce thermal effects, let's suppose that a trivial beam splitter has its two probabilities $p_{1}$ and $1-p_{1}$, depend on temperature $T$ according to a positive energy difference $\Delta E_{1}$ and temperature $T$ via the Boltzmann factor

$$
\begin{aligned}
& p_{1} \propto \exp \left(\frac{-\Delta E_{1}}{k_{b} T}\right), \\
& 1-p_{1} \propto \exp \left(\frac{+\Delta E_{1}}{k_{b} T}\right) .
\end{aligned}
$$

The proportionality can be determined by the requirement that $p_{1}$ and $1-p_{1}$ add to 1 . So we divide by the sum of the right hand sides to get the

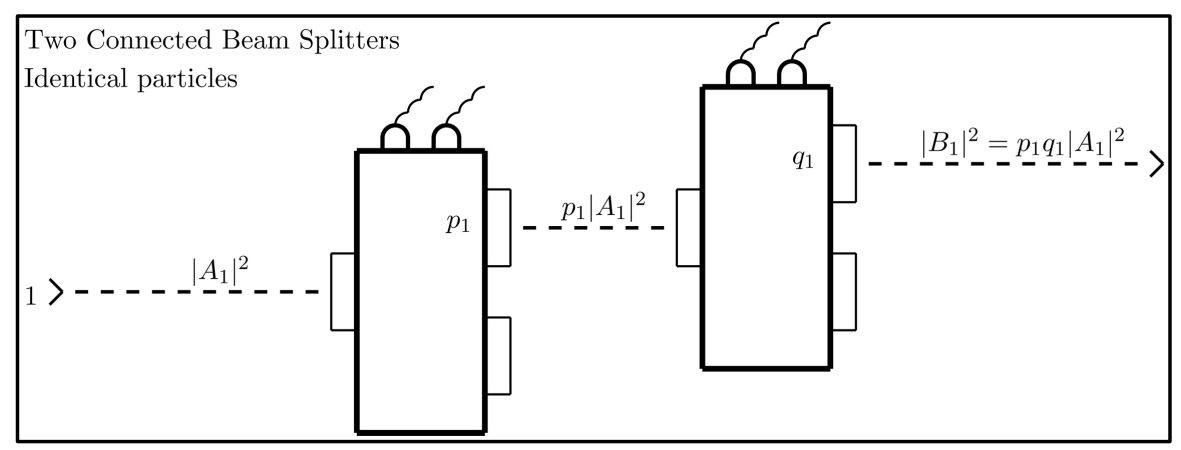

Figure 2. Two trivial beam splitters connected together. Particles of type 1 arrive at rate $\left|A_{1}\right|^{2}$. The beam splitters have probability $p_{1}$ and $q_{1}$ so the overall probability is the product $p_{1} q_{1}$ and the output beam has a rate of $\left|B_{1}\right|^{2}=p_{1} q_{1}\left|A_{1}\right|^{2}$. 
probabilities:

$$
\begin{aligned}
& p_{1}=\exp \left(\frac{-\Delta E_{1}}{k_{b} T}\right) /\left(\exp \left(\frac{-\Delta E_{1}}{k_{b} T}\right)+\exp \left(\frac{+\Delta E_{1}}{k_{b} T}\right)\right), \\
& 1-p_{1}=\exp \left(\frac{+\Delta E_{1}}{k_{b} T}\right) /\left(\exp \left(\frac{-\Delta E_{1}}{k_{b} T}\right)+\exp \left(\frac{+\Delta E_{1}}{k_{b} T}\right)\right) .
\end{aligned}
$$

In the high temperature limit $k_{b} T \gg \Delta E_{1}$, the probabilities approach $p_{1}=1-p_{1}=1 / 2$.

At the low temperature limit, the probabilities go to $p_{1}=1, p_{0}=0$ and near zero, they are approximately

$$
\begin{aligned}
& p_{1} \approx \exp \left(\frac{-2 \Delta E_{1}}{k_{b} T}\right), \\
& p_{2} \approx 1-\exp \left(\frac{-2 \Delta E_{1}}{k_{b} T}\right) .
\end{aligned}
$$

In this cold limit, putting $n$ beam splitters together by taking the exit ports to the input port of the next beam splitter will give a final upper exit port probability of $p_{1}^{n}$ and this corresponds to decreasing the temperature from $T$ to $T / n$ :

$$
\left(p_{1}\right)^{n} \approx\left(\exp \left(\frac{-2 \Delta E_{1}}{k_{b} T}\right)\right)^{n}=\exp \left(\frac{-2 \Delta E_{1}}{k_{b} T / n}\right)
$$

Thus connecting identical thermal beam splitters has the effect of reducing the temperature. This temperature effect will also work in the quantum situation.

\subsection{Classical Two Particle Beam Splitters}

The reader may be relieved to read that now we consider beam splitters that act on a particle beam with two kinds of particles, type 1 and type 2 . The two particles arrive with rates $\left|A_{1}\right|^{2}$ and $\left|A_{2}\right|^{2}$ and the probabilities that they leave via the upper exits are $p_{1}$ and $p_{2}$. If the output of the beam splitter is sent to another identical beam splitter, the probabilities will square so that

$$
\begin{aligned}
& \left|B_{1}\right|^{2}=p_{1} p_{1}\left|A_{1}\right|^{2}=\left(p_{1}\right)^{2}\left|A_{1}\right|^{2}, \\
& \left|B_{2}\right|^{2}=p_{2} p_{2}\left|A_{2}\right|^{2}=\left(p_{2}\right)^{2}\left|A_{2}\right|^{2} .
\end{aligned}
$$

We can rewrite the above equations in $2 \times 2$ diagonal matrix form:

$$
\left(\begin{array}{cc}
\left|B_{1}\right|^{2} & 0 \\
0 & \left|B_{2}\right|^{2}
\end{array}\right)=\left(\begin{array}{cc}
p_{1} & 0 \\
0 & p_{2}
\end{array}\right)\left(\begin{array}{cc}
p_{1} & 0 \\
0 & p_{2}
\end{array}\right)\left(\begin{array}{cc}
\left|A_{1}\right|^{2} & 0 \\
0 & \left|A_{2}\right|^{2}
\end{array}\right)
$$

See Figure 3.

The reader may notice that we could have put the $\left|B_{j}\right|^{2}$ and $\left|A_{j}\right|^{2}$ numbers into vectors instead of matrices. We're doing it with matrices so that our presentation will be compatible with the density matrices of quantum mechanics. For arguments supporting density matrices as a superior way of representing quantum states see [8]. 


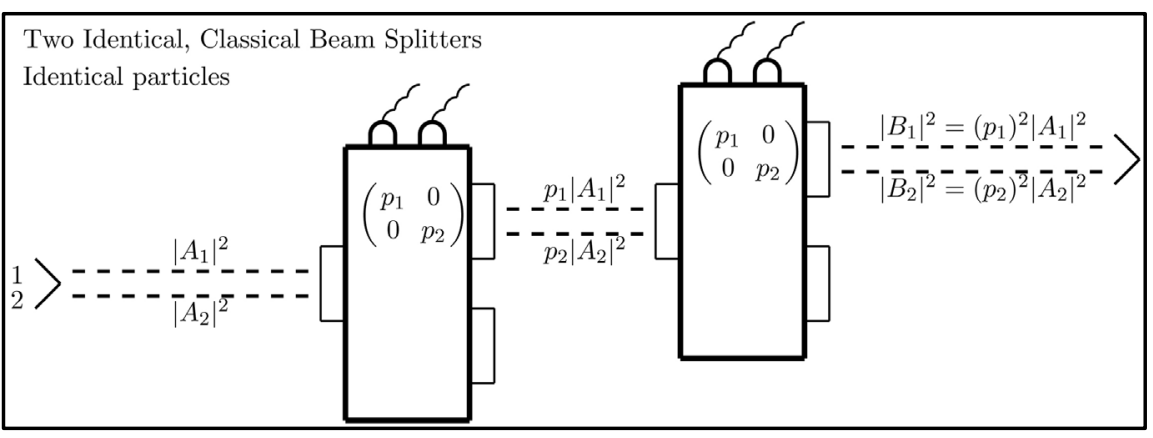

Figure 3. Two identical, classical beam splitters connected together. Particles of type 1 and 2 arrive mixed together in a single beam with rates $\left|A_{1}\right|^{2}$ and $\left|A_{2}\right|^{2}$. The beam splitters have probability $p_{1}$ and $p_{2}$ for the two types so their overall probabilities are $\left(p_{1}\right)^{2}$ and $\left(p_{2}\right)^{2}$. This can be expressed with matrix multiplication.

With the probabilities for the two particle types being $p_{1}$ and $p_{2}$, the corresponding probabilities for the lower exit will be $1-p_{1}$ and $1-p_{2}$. This is represented by a matrix with those numbers on the diagonal. So if we recombine the lower and upper exit ports the resulting experiment will be represented by the sum of the two matrices which is the unit matrix:

$$
\left(\begin{array}{cc}
p_{1} & 0 \\
0 & p_{2}
\end{array}\right)+\left(\begin{array}{cc}
1-p_{1} & 0 \\
0 & 1-p_{2}
\end{array}\right)=\left(\begin{array}{ll}
1 & 0 \\
0 & 1
\end{array}\right)=\hat{1}
$$

The unit matrix has no effect on particle rates so, as expected, the effect of recombining the two outputs of a beam splitter is to give back a beam identical to the original.

\section{Quantum Beam Splitters}

In the classical situation, particles do not interfere with each other so we can use rates such as $\left|A_{j}\right|^{2}$ to completely describe a mixture. For such a situation, the rates add the same way that probabilities do. For example, if we have two beams each with the same rate $\left|A_{1}\right|^{2}$, combining the beams will give a beam with twice that rate $2\left|A_{1}\right|^{2}$.

In the classical situation, we used real diagonal matrices to represent the beam splitters and the product of two real diagonal matrices is also real diagonal so real diagonal matrices were sufficient to represent a single beam splitters as well as a connected series of beam splitters. For the quantum case, an individual beam splitter will be represented by an Hermitian matrix and products of these matrices are more general than Hermitian.

\subsection{Pauli Spin Matrices}

Most of this paper is restricted to the subject of beams with only two particle types. For quantum mechanics the standard example is su(2) spin-1/2 where the two particle types are typically taken to be spin-up which we will label with $+Z$ and spin-down which we will label as $-z$. There being nothing special about the 
$z$ direction, we can also consider spin-1/2 measured in other directions such as $\pm x, \pm y$ or for spin measured in a general direction $u$.

A matrix $\rho_{+z}$ for measuring spin-1/2 in the $+z$ direction will give spin-up in the upper exit. The reverse measurement $\rho_{-z}$, for spin in the $-z$ direction will give spin-down in the upper exit. The (classical or quantum) matrices for these measurements are:

$$
\begin{aligned}
& \rho_{+z}=\left(\begin{array}{ll}
1 & 0 \\
0 & 0
\end{array}\right), \\
& \rho_{-z}=\left(\begin{array}{ll}
0 & 0 \\
0 & 1
\end{array}\right) .
\end{aligned}
$$

The above matrices are idempotents, that is, they are not changed by squaring $\left(\rho_{ \pm z}\right)^{2}=\rho_{ \pm z}$. This is an important feature of spin measurements; repeating the measurement gives the same result. And the two matrices sum to $\hat{1}$; that is, spin-up and spin-down account for all the possible measurements (exit ports). We require that the matrices for spin-1/2 measurements in any other direction have these features.

Consider a general idempotent $2 \times 2$ complex matrix where we've included a factor of $1 / 2$ for later convenience:

$$
\rho(a, b, c, d)=\frac{1}{2}\left(\begin{array}{ll}
a & b \\
c & d
\end{array}\right) \text {, where } \rho^{2}=\rho .
$$

This gives four equations:

$$
\begin{aligned}
& a / 2=\left(a^{2}+b c\right) / 4, \\
& b / 2=b(a+d) / 4, \\
& c / 2=c(a+d) / 4, \\
& d / 2=\left(d^{2}+b c\right) / 4 .
\end{aligned}
$$

If we let $b=c=0$, we would have the classical case. Dividing the middle two of the above four equations by $b$ or $c$ implies that $a+d=2$. Applying this to the sum of the first and fourth equation we get $1=\left(a^{2}+d^{2}+2 b c\right) / 4$.

Now consider spin measurements on the $z=0$ plane, that is, the equator of the sphere $(\cos (\theta), \sin (\theta), 0)$. These directions are equidistant from the $\pm Z=(0,0, \pm 1)$ directions so we expect to get equal particle rates when we insert one of these measurements between two $\rho_{+z}$ or two $\rho_{-z}$ measurements. Computing we find

$$
\begin{aligned}
& \rho_{+z} \rho(a, b, c, d) \rho_{+z}=(a / 2) \rho_{+z}, \\
& \rho_{-z} \rho(a, b, c, d) \rho_{-z}=(d / 2) \rho_{-z} .
\end{aligned}
$$

And so for $\rho(a, b, c, d)$ to have the same effect on spin-up as it does on spin-down, we must have that $a=d$. Together with $a+d=2$ this shows that $a=d=1$ giving

$$
\begin{aligned}
& \rho_{+z} \rho(a, b, c, d) \rho_{+z}=(1 / 2) \rho_{+z}, \\
& \rho_{-z} \rho(a, b, c, d) \rho_{-z}=(1 / 2) \rho_{-z} .
\end{aligned}
$$


Having solved for $a$ and $d$ we find that $c=1 / b$. This gives the general solution for matrices for spin measurements on the $z=0$ plane as

$$
\rho(b)=\frac{1}{2}\left(\begin{array}{cc}
1 & b \\
b^{-1} & 1
\end{array}\right) .
$$

We need different $b$ values for spin-1/2 measurements in the $+x$ and $+y$ directions. Call these $b_{x}$ and $b_{y}$.

Consider a sequence of measurements that begin with spin in the $+x$ direction, then $+y$, and finally $+x$. This complex measurement will end up as a $(1 / 2)$ multiple of $\rho_{+x}$ just as we found in Equation (12). Calculating we have

$$
\begin{aligned}
& \rho_{+x} \rho_{+y} \rho_{+x}=(1 / 2) \rho_{+x}, \\
& \frac{1}{2}\left(\begin{array}{cc}
1 & b_{x} \\
b_{x}^{-1} & 1
\end{array}\right) \frac{1}{2}\left(\begin{array}{cc}
1 & b_{y} \\
b_{y}^{-1} & 1
\end{array}\right) \frac{1}{2}\left(\begin{array}{cc}
1 & b_{x} \\
b_{x}^{-1} & 1
\end{array}\right)=(1 / 2) \frac{1}{2}\left(\begin{array}{cc}
1 & b_{x} \\
b_{x}^{-1} & 1
\end{array}\right) .
\end{aligned}
$$

Multiplying out the matrices gives four equations, each of which is equivalent to $2=2+b_{x} / b_{y}+b_{y} / b_{x}$ or

$$
b_{x}^{2}+b_{y}^{2}=0 \text { or } b_{x}=i b_{y} .
$$

This shows that in order to model 3 dimensions, we will need complex numbers.

So for any complex number $b=b_{x}=i b_{y}$, with $|b|$ not zero, we obtain three projection operators;

$$
\begin{aligned}
& \rho_{ \pm x}=\frac{1}{2}\left(\begin{array}{cc}
1 & \pm b \\
\pm b^{-1} & 1
\end{array}\right), \\
& \rho_{ \pm y}=\frac{1}{2}\left(\begin{array}{cc}
1 & \mp i b \\
\pm i b^{-1} & 1
\end{array}\right), \\
& \rho_{ \pm z}=\frac{1}{2}\left(\begin{array}{cc} 
\pm 1 & 0 \\
0 & 1 \mp 1
\end{array}\right) .
\end{aligned}
$$

The above projection operators are of the form $\rho_{ \pm u}=0.5\left(\hat{1} \pm \sigma_{u}(b)\right)$ where $\sigma_{u}(b)$ are three matrices:

$$
\begin{aligned}
& \sigma_{x}(b)=\left(\begin{array}{cc}
0 & b \\
b^{-1} & 0
\end{array}\right), \\
& \sigma_{y}(b)=\left(\begin{array}{cc}
0 & -i b \\
i b^{-1} & 0
\end{array}\right), \\
& \sigma_{z}(b)=\left(\begin{array}{cc}
1 & 0 \\
0 & -1
\end{array}\right) .
\end{aligned}
$$

These matrices each square to $\hat{1}$ and anti-commute:

$$
\begin{aligned}
& \sigma_{x}(b)^{2}=\sigma_{y}(b)^{2}=\sigma_{z}(b)^{2}=1, \\
& \sigma_{x}(b) \sigma_{y}(b)=-\sigma_{y}(b) \sigma_{x}(b), \\
& \sigma_{y}(b) \sigma_{z}(b)=-\sigma_{z}(b) \sigma_{y}(b), \\
& \sigma_{z}(b) \sigma_{x}(b)=-\sigma_{x}(b) \sigma_{z}(b) .
\end{aligned}
$$


With the choice $b=1$, they are called the "Pauli spin matrices" and are Hermitian. From here on we will use them:

$$
\begin{aligned}
& \sigma_{x}=\left(\begin{array}{ll}
0 & 1 \\
1 & 0
\end{array}\right), \\
& \sigma_{y}=\left(\begin{array}{cc}
0 & -i \\
i & 0
\end{array}\right), \\
& \sigma_{z}=\left(\begin{array}{cc}
1 & 0 \\
0 & -1
\end{array}\right) .
\end{aligned}
$$

Any Hermitian $2 \times 2$ matrix $H$ can be written using $\hat{1}$ and the Pauli spin matrices as a basis over the real numbers:

$$
\begin{aligned}
H & =h_{0}\left(\begin{array}{ll}
1 & 0 \\
0 & 1
\end{array}\right)+h_{1}\left(\begin{array}{ll}
0 & 1 \\
1 & 0
\end{array}\right)+h_{2}\left(\begin{array}{cc}
0 & -i \\
i & 0
\end{array}\right)+h_{3}\left(\begin{array}{cc}
1 & 0 \\
0 & -1
\end{array}\right), \\
& =h_{0} \hat{1}+h_{1} \sigma_{x}+h_{2} \sigma_{y}+h_{3} \sigma_{z} .
\end{aligned}
$$

where $h_{j}$ are four real numbers. Pure or mixed density matrices will have $h_{0}=1 / 2$ so that the trace is 1 . The pure and mixed cases are distinguished by $h_{1}^{2}+h_{2}^{2}+h_{3}^{2}=1 / 4$ and $h_{1}^{2}+h_{2}^{2}+h_{3}^{2}<1 / 4$, respectively. At the infinite temperature limit, $h_{1}=h_{2}=h_{3}=0$.

With the Pauli spin matrices, the projection operators for spin in the $\pm x$, $\pm y$ and $\pm z$ directions are defined as:

$$
\begin{aligned}
& \rho_{ \pm x}=\left(\hat{1} \pm \sigma_{x}\right) / 2, \\
& \rho_{ \pm y}=\left(\hat{1} \pm \sigma_{y}\right) / 2, \\
& \rho_{ \pm z}=\left(\hat{1} \pm \sigma_{z}\right) / 2 .
\end{aligned}
$$

One can also define a spin-1/2 matrix for spin in other directions. Given a unit vector $u=\left(u_{x}, u_{y}, u_{z}\right)$, the spin matrix is:

$$
\begin{aligned}
\sigma_{u} & =u_{x} \sigma_{x}+u_{y} \sigma_{y}+u_{z} \sigma_{z}, \\
& =\left(\begin{array}{cc}
u_{z} & u_{x}-i u_{y} \\
u_{x}+i u_{y} & -u_{z}
\end{array}\right) .
\end{aligned}
$$

Like the usual Pauli spin matrices, $\sigma_{u}$ squares to $\hat{1}$ and can be used to define the projection operator for spin-1/2 in the $u$ direction by $\rho_{+u}=\left(\hat{1}+\sigma_{u}\right) / 2$.

The assumption that observables are Hermitian is often included as an axiom of quantum mechanics. The justification is that observables should be real numbers and, since Hermitian matrices have real eigenvalues, they are a natural choice. As hinted at in Equation (17), one can modify Hermitian matrices by multiplying a column by a nonzero complex number $b$ and the corresponding row by $b^{-1}$. This transformation is a isomorphism in that it preserves matrix multiplication and addition. The transformation, in general, takes Hermitian matrices to non Hermitian matrices but leaves their eigenvalues unchanged and only slightly modifies their eigenvectors. So we see that while it is convenient for quantum mechanics to use Hermitian matrices it is not necessary. 
Two Hermitian matrices $H$ and $K$ have a product $H K$ that is not in general Hermitian. The product will be Hermitian if and only if the two matrices commute. We can write $H K=(H K+K H) / 2+(H K-K H) / 2$ and split the product into an Hermitian part $(H K+K H) / 2$ and an anti-Hermitian part $(H K-K H) / 2$. Multiplying the anti-Hermitian part by $i$ gives the Hermitian matrix $i(H K-K H) / 2$. For matrices that represent beam splitters, the non commutative case corresponds to the physical situation where the order of measurements has an effect as we discuss in Subsection 3.2.

A perfect beam splitter for spin-1/2 in the $+u$ direction is represented by the matrix $\rho_{+u}=\left(\hat{1}+\sigma_{u}\right) / 2$. The particles that take the lower exit will be spin- $1 / 2$ in the $-u$ direction so that port will be represented by the matrix $\rho_{-u}=\left(\hat{1}-\sigma_{u}\right) / 2$. These two matrices sum to unity:

$$
\rho_{+u}+\rho_{-u}=\left(\hat{1}+\sigma_{u}\right) / 2+\left(\hat{1}-\sigma_{u}\right) / 2=\hat{1},
$$

so, as in the classical case, combining the outputs of a single perfect beam splitter results in an experiment that has no effect on the beam.

Recombining the two exits has the effect of canceling the measurement. This behavior may seem contradictory to the concept that measurements affect quantum systems. Perhaps beam splitters can be better described as devices that split waves rather than devices that make measurements on particles. The traditional notation we use in this paper promotes the "separation fallacy" well described in [9]. To make a measurement requires something to absorb the particle and be permanently altered such as a photographic plate. Beam splitters do not measure, they allow measurements. Until measurement the particle is just a wave and can be recombined the same way that the electric and magnetic fields of a light wave can be recombined.

\subsection{Geometric Phase/Perfect Beam Splitter Calculations}

Suppose we have three perfect quantum beam splitters, two for spin-1/2 in the $+x$ direction and one for spin- $1 / 2$ in the $+z$ direction. We connect them up so that a particle is measured first for spin in $+x$ direction, then spin in the $+z$ direction and finally spin in the $+x$ direction. The matrix corresponding to the three consecutive measurements is given by the product of the matrices. Computing with the above matrix definitions we have:

$$
\rho_{+x+z+x}=\rho_{+x} \rho_{+z} \rho_{+x}=\frac{1}{2} \rho_{+x}=(\sqrt{1 / 2})^{2} \rho_{+x} .
$$

Compared with a single measurement of spin-1/2 in the $+X$ direction, the compound measurement is decreased by a factor $(\sqrt{1 / 2})^{2}$. In the second equality, the $1 / 2$ indicates that the compound measurement has a reduction in the particle rate of $1 / 2$, i.e. $\left|B_{1}\right|^{2}=(1 / 2)\left|A_{1}\right|^{2}$. The rate reduction is the result of particles having to navigate the change in the measurements between $+Z$ and $+x$. But there are two such navigation changes so each is having an effect of $\sqrt{1 / 2}$ as shown in the third equality. 
Suppose we have four perfect quantum beam splitters, two for spin-1/2 in the $+z$ direction $\left(\right.$ i.e. $\rho_{+z}$ ) and one each for spin in the $+x$ and $+y$ direction (i.e. $\rho_{+x}$ and $\left.\rho_{+y}\right)$. We connect the beam splitters so a particle first enters one of the $+Z$ spin-1/2 measurements, then if it reaches the upper exit it is made to enter a beam splitter for the $+x$ spin- $1 / 2$ measurement, then the $+y$ spin- $1 / 2$ measurement and finally it enters the second $+Z$ spin- $1 / 2$ measurement. Computing, we find:

$$
\rho_{+z+x+y+z}=\rho_{+z} \rho_{+y} \rho_{+x} \rho_{+z}=\frac{1-i}{4} \rho_{+z}=\mathrm{e}^{i(1 / 2)(-\pi / 2)}(\sqrt{1 / 2})^{3} \rho_{+z}
$$

The final equality in the above shows that the results of connecting the four beam splitters is a complex multiple of the last (or first) beam splitter, and so is not Hermitian. There are a number of observations to make here. The first is that since the initial and final beam splitters are identical, the compound beam splitter is a complex multiple of that initial/final beam splitter. This is a general fact about products of primitive idempotent matrices that begin and end with the same primitive idempotent matrix.

A second observation is the factor $(\sqrt{1 / 2})^{3}$. As with Equation (24), this comes from the particles having to navigate three changes in measurement: $+Z$ to $+x$, then from $+x$ to $+y$, and finally from $+y$ to $+z$. More generally, if two perfect quantum beam splitters have directions that differ by $\theta$ degrees, combining them will reduce the amplitude by $\sqrt{(1+\cos (\theta)) / 2}$. This can be verified by putting $u=(\sin (\theta), 0, \cos (\theta))$ and computing

$$
\rho_{+z+u+z}=\rho_{+z} \rho_{+u} \rho_{+z}=\left(\sqrt{\frac{1+\cos (\theta)}{2}}\right)^{2} \rho_{+z} .
$$

That is, there are two transitions, $+z$ to $+x$ and then to $+z$ so the factor appears squared.

The last observation on Equation (25) is in regard to the complex phase $\exp (i(1 / 2)(-\pi / 2))$. This is called a "geometric phase" as it depends on the geometric area included in the path. Our path goes from $+z$ to $+x$ to $+y$ and then back to $+z$. This is one octant of the surface of the sphere. The total surface of the sphere is $4 \pi$ steradians so an octant is an eighth of this which gives the factor $4 \pi / 8=\pi / 2$. The area is oriented and our path is traversed in the negative direction so the $\pi / 2$ takes a minus sign giving $(-\pi / 2)$. The remaining factor of $1 / 2$ has to do with this being a path in the spin of a spin-1/2 particle. The factor $1 / 2$ implies that when the path is taken around the equator (or any great circle), the quantum phase will be $(1 / 2)(2 \pi)=\pi$ so the particle will get $\mathrm{e}^{i \pi}=-1$. This is the -1 one obtains by rotating a fermion through $360^{\circ}$. A good exercise for students is to find the "remaining factor" for the 1, 0 and -1 cases of spin-1. For one of these cases, they may find it useful to read Subsection 3.4.

Together, these three observations allow quick computation of the matrix corresponding to compound perfect quantum measurements in spin-1/2. For example, suppose the path includes one face of an icosahedron. Letting $\theta \approx 65.435^{\circ}$ be the angle between two adjacent points on an icosahedron, the 
product of the matrices will include a factor of $((1+\cos (\theta)) / 2)^{3 / 2}$ for the three changes in spin direction. Since the icosahedron has 20 faces, the geometric phase will be $\exp (i(1 / 2)( \pm 4 \pi / 20))$ where the sign depends on which direction is taken.

\subsection{Quantum Amplitudes}

We now rewrite our particle rates $\left|A_{j}\right|^{2}$ in terms of amplitudes $A_{j}$ as is traditional for quantum mechanics. We begin with a classical beam splitter acting on a beam with two particle types. From Subsection 2.2, the incoming particle rates $\left|A_{j}\right|^{2}$ are related to the exit particle rates $\left|B_{k}\right|^{2}$ by

$$
\left(\begin{array}{cc}
\left|B_{1}\right|^{2} & 0 \\
0 & \left|B_{2}\right|^{2}
\end{array}\right)=\left(\begin{array}{cc}
q_{1} & 0 \\
0 & q_{2}
\end{array}\right)\left(\begin{array}{cc}
\left|A_{1}\right|^{2} & 0 \\
0 & \left|A_{2}\right|^{2}
\end{array}\right)
$$

where $q_{j}$ are probabilities for the passage of the two particle types. The two particle rate matrices are treated the same way; we'll discuss the $\left|A_{j}\right|^{2}$ matrix. We replace the matrices as follows:

$$
\left(\begin{array}{cc}
\left|A_{1}\right|^{2} & 0 \\
0 & \left|A_{2}\right|^{2}
\end{array}\right)=\left(\begin{array}{c}
A_{1} \\
0
\end{array}\right)\left(\begin{array}{cc}
A_{1}^{*} & 0
\end{array}\right)+\left(\begin{array}{c}
0 \\
A_{2}
\end{array}\right)\left(\begin{array}{ll}
0 & A_{2}^{*}
\end{array}\right) .
$$

This factors an entry in the rate matrix into a product of two matrices, one $2 \times 1$ and the other $1 \times 2$. These are called bras and kets. The kets are:

$$
\begin{aligned}
& \left(\begin{array}{c}
A_{1} \\
0
\end{array}\right)=A_{1}\left(\begin{array}{l}
1 \\
0
\end{array}\right)=A_{1}|+z\rangle, \\
& \left(\begin{array}{l}
0 \\
A_{2}
\end{array}\right)=A_{2}\left(\begin{array}{l}
0 \\
1
\end{array}\right)=A_{2}|-z\rangle,
\end{aligned}
$$

while the bras are:

$$
\begin{aligned}
& \left(\begin{array}{ll}
A_{1}^{*} & 0
\end{array}\right)=A_{1}^{*}\left(\begin{array}{ll}
1 & 0
\end{array}\right)=A_{1}^{*}\langle+Z|, \\
& \left(\begin{array}{ll}
0 & A_{2}^{*}
\end{array}\right)=A_{2}^{*}\left(\begin{array}{ll}
0 & 1
\end{array}\right)=A_{2}^{*}\langle-z| .
\end{aligned}
$$

In most quantum mechanics textbooks, our notation $| \pm z\rangle$ is replaced with $|\uparrow\rangle$ and $|\downarrow\rangle$. We will use the $\pm z$ notation as we are concerned with other spin measurements in other directions besides $z$.

In the bra ket notation, the particle rates are a product of bras and kets:

$$
\begin{aligned}
& \left|A_{1}\right|^{2}=A_{1}\left\langle+z\left|A_{1}^{*}\right|+z\right\rangle=\left|A_{1}\right|^{2}\langle+z \mid+z\rangle, \\
& \left|A_{2}\right|^{2}=A_{2}\left\langle-z\left|A_{2}^{*}\right|-z\right\rangle=\left|A_{2}\right|^{2}\langle-z \mid-z\rangle .
\end{aligned}
$$

The above multiplications give a calculation which is bracketed " \langle\rangle ”. Hence the \langle| is called a bra and the |\rangle is called a ket. In this notation, the matrix of particle rates in Equation (28) becomes:

$$
\begin{aligned}
\left(\begin{array}{cc}
\left|A_{1}\right|^{2} & 0 \\
0 & \left|A_{2}\right|^{2}
\end{array}\right) & =A_{1}|+z\rangle A_{1}^{*}\left\langle+z\left|+A_{2}\right|-z\right\rangle A_{2}^{*}\langle-z|, \\
& =\left|A_{1}\right|^{2}|+z\rangle\left\langle+\left.z|+| A_{2}\right|^{2} \mid-z\right\rangle\langle-z| .
\end{aligned}
$$


Splitting the rate matrix into $+Z$ and $-Z$ components allows us to rewrite Equation (27) which deals with the particle rates $\left|B_{j}\right|^{2}$ and $\left|A_{j}\right|^{2}$ into a pair of equations that relate the amplitudes $A_{j}| \pm z\rangle$ and $B_{j}| \pm z\rangle$ :

$$
\begin{aligned}
& B_{1}|+z\rangle=\left(\begin{array}{cc}
q_{1} & 0 \\
0 & q_{2}
\end{array}\right) A_{1}|+z\rangle, \\
& B_{2}|-z\rangle=\left(\begin{array}{cc}
q_{1} & 0 \\
0 & q_{2}
\end{array}\right) A_{2}|-z\rangle,
\end{aligned}
$$

This shows that classical beams, like the quantum case, can naturally be analyzed in terms of amplitudes instead of probabilities.

\subsection{Berry-Pancharatnam Phases}

Geometric phases are related to the "Berry-Pancharatnam phases" that a quantum state receives when it is adiabatically sent through a cycle. In the last example of Subsection 3.2, the cycle is from spin-1/2 in the $+z$ direction, through a sequence of spin measurements in other directions and then back to the $+Z$ direction.

To make the cycle adiabatic, we insert intermediate spin measurements between two consecutive pairs of measurements. For example, between the $+Z$ and $+x$ measurement, we would insert a large number $N$ of spin-1/2 measurements in the directions $u(n)=(\sin ((n / N) \pi / 2), 0, \cos ((n / N) \pi / 2))$ so that consecutive measurements differ in direction by only $(1 / N) \pi / 2$ radians. The intermediate measurements are on the great circle route between the $+z$ and $+x$ directions. If we deviated from this path, the area enclosed by the cycle could change and this would change the geometric phase.

Any two points on the surface of the sphere define a great circle route unless those two points are on opposing ends of a diameter (antipodal). For a unit vector $u$, the points $u$ and $-u$ are on the opposite ends of a diameter and we have

$$
\rho_{u} \rho_{-u}=0 .
$$

That is, the projection operators for opposite spin measurements annihilate each other and there is no unique great circle route to make the transition adiabatic.

The usual introduction to spin-1/2 uses raising and lowering operators. For the spin-1/2 case, these are operators that negate the direction of a quantum state (amplitude), for example the raising operator for spin-1/2 takes the spin-down state to a spin-up state. More general raising and lowering operators change a state from one spin state to an orthogonal spin state and again the projection operators annihilate.

The absence of a great circle route between annihilating spin projection operators means that there is no natural phase that can be assigned for such an operator. For example, the raising operator for spin-1/2 can be written as:

$$
\rho_{+}(\theta)=\left(\begin{array}{cc}
0 & \exp (-i \theta) \\
0 & 0
\end{array}\right)
$$


where $\theta$ is any angle. In the standard presentation of raising operators, $\theta$ is chosen as zero. We can obtain the general case by making a choice for the intermediate measurement along the equator of the sphere at the point $u(\theta)=(\cos (\theta), \sin (\theta), 0)$ so that

$$
\rho_{+u}(\theta)=\left(1+\cos (\theta) \sigma_{x}+\sin (\theta) \sigma_{y}\right) / 2 .
$$

Then the arbitrary phased raising operator can be written as a product of projection operators:

$$
\left(\begin{array}{cc}
0 & \exp (-i \theta) \\
0 & 0
\end{array}\right)=2 \rho_{+z} \rho_{+u}(\theta) \rho_{-z}
$$

where the factor of 2 is included due to the $\sqrt{1 / 2}$ loss in amplitude in the two transitions. The standard raising operator is obtained by choosing the intermediate measurement as $\rho_{+x}=\left(1+\sigma_{x}\right) / 2$.

\subsection{Quantum Temperatures}

Temperatures work the same in the quantum situation as they do in the classical. Using the classical numbers from Equation (1), a quantum beam splitter acting on a beam with only a single particle type will be identical to the classical case. To make it more interesting we can consider such a device acting on a beam with two particle types. Consider a measurement of spin- $1 / 2$ in the $+z$ direction. A perfect measurement (temperature zero) is given by $\left(1+\sigma_{z}\right) / 2$. At finite temperatures some of the spin-up particles end up in the lower exit and some of the spin-down particles take the upper exit. Using $+\Delta E$ for the first particle type and $-\Delta E$ for the second we have a matrix for the upper exit port:

$$
\rho(T)=\left(\begin{array}{cc}
\exp \left(\frac{+\Delta E}{k_{b} T}\right) & 0 \\
0 & \exp \left(\frac{-\Delta E}{k_{b} T}\right)
\end{array}\right) /\left(\exp \left(\frac{+\Delta E}{k_{b} T}\right)+\exp \left(\frac{-\Delta E}{k_{b} T}\right)\right)
$$

In the low temperature limit, and choosing to keep the sum of the probabilities 1, these become

$$
\rho(T) \approx\left(\begin{array}{cc}
1-\exp \left(\frac{-2 \Delta E}{k_{b} T}\right) & 0 \\
0 & \exp \left(\frac{-2 \Delta E}{k_{b} T}\right)
\end{array}\right),
$$

similar to the classical result in Equation (4).

We can connect $n$ of these experiments together so that the upper exit of one beam splitter goes to the entrance of the next; the composite experiment is modeled by the $n$ power of the matrix $\rho(T)$ :

$$
(\rho(T))^{n} \approx\left(\begin{array}{cc}
1-\exp \left(\frac{-2 \Delta E}{k_{b} T / n}\right) & 0 \\
0 & \exp \left(\frac{-2 \Delta E}{k_{b} T / n}\right)
\end{array}\right)
$$


The above is diagonal, as would be obtained for a classical thermal two particle beam splitter. To get off diagonal elements we need to consider spin in directions other than $\pm z$. Our calculations have been for spin-1/2 in the $+z$ direction but that is not a special direction so we can generalize to the $+u$ direction. To do that, we need to rewrite the above in the Pauli spin matrix basis. We find

$$
(\rho(T))^{n} \approx\left(\hat{1}+\left(1-\exp \left(\frac{-2 \Delta E}{k_{b} T / n}\right)\right) \sigma_{+u}\right) / 2 .
$$

where $\sigma_{z}$ has been replaced with $\sigma_{+u}$ to give the general spin-1/2 case.

Squaring a low temperature matrix $\rho(T)$ approximately gives the matrix for an even lower temperature $\rho(T / 2)$. For general temperatures, the squaring does not necessarily divide the temperature by two but (other than the high temperature limit) it does reduce the temperature. The high temperature limit has probabilities $1 / 2$ :

$$
\rho\left(T \gg \Delta E / k_{B}\right) \approx\left(\begin{array}{cc}
1 / 2 & 0 \\
0 & 1 / 2
\end{array}\right) .
$$

Since the matrices for the upper and lower exits must sum to $\hat{1}$, the lower exit matrix must be identical to the above. Squaring the above matrix gives a matrix with $1 / 4 \mathrm{~s}$ on the diagonal instead of $1 / 2 \mathrm{~s}$ but the matrix must still correspond to the same high temperature limit so we need to multiply the matrix by 2 . This "renormalization" was (approximately) unnecessary at very low temperatures. At higher temperatures, one can maintain the trace as one by dividing the matrix by the trace after squaring. The trace of the above matrix is $1 / 2$ so dividing by $1 / 2$ will renormalize it. Density matrices are beyond the scope of this paper however we note that requiring our matrices to have trace 1 makes them mathematically identical to "mixed density matrices" and when density matrices are used in statistical physics, squaring and renormalization is a method used to reduce their temperature [10].

\subsection{Superselection Sectors}

Suppose we have a beam of spin-up electrons and we split it with a beam splitter measuring spin-1/2 in the $u=\left(u_{x}, u_{y}, u_{z}\right)$ direction. The entering beam is represented by a ket that is pure spin-up:

$$
|+z\rangle=\left(\begin{array}{l}
1 \\
0
\end{array}\right)
$$

and the matrix representing the measurement is

$$
\rho_{+u}=\left(\hat{1}+\sigma_{u}\right) / 2=\frac{1}{2}\left(\begin{array}{cc}
1+u_{z} & u_{x}-i u_{y} \\
u_{x}+i u_{y} & 1-u_{z}
\end{array}\right) .
$$

Performing the matrix multiplication $\rho_{+u}|+z\rangle$, the exiting beam is represented by a ket for spin- $1 / 2$ in the $+u$ direction: 


$$
|+u\rangle=\frac{1}{2}\left(\begin{array}{c}
1+u_{z} \\
u_{x}+i u_{y}
\end{array}\right)=\frac{1+u_{z}}{2}|+z\rangle+\frac{u_{x}+i u_{y}}{2}|-z\rangle .
$$

The above shows that it is physically possible to begin with a beam of pure spin-up and from it make a beam that is a "linear superposition" of spin-up and spin-down. This is accomplished by arranging for the matrix $\rho_{+u}$ to have non zero off diagonal elements. That is, we will get a linear superposition provided $u_{x}$ or $u_{y}$ is non zero.

It's natural for students to imagine that it's possible to create a superposition of any quantum states, given that physicists talk about superposition of states consisting of a live and dead cat. But in fact, not all quantum superpositions can be created; superpositions are limited by "superselection sectors" and this is what we will discuss in this subsection. Superselection sectors can be attributed to two subjects beyond the scope of this paper, symmetry and decoherence so we will provide only an outline of the ideas. The subject is explained in [11]; we will explore the subject from the point of view of binary measurements.

Our starting point is to assume, as Julian Schwinger did, that given a "complete set of commuting observables" to define a quantum state, it is possible to define a beam splitter whose upper exit passes only that particular state, for example, spin-up. The spin-1/2 symmetry defines states for all the possible directions $u$ so we can use intermediate states to obtain a superposition between spin-up and spin-down as was illustrated in Equation (45).

We will now consider a binary measurement on a particle beam that contains electrons and neutrinos. There are no intermediate quantum states between the electron and neutrino so there is no way for us to use our spin-1/2 example to convert an electron beam into a beam that is a superposition of electron and neutrino. This is the most common case for "internal" symmetries, however, the particle "generations" are an exception and in fact, the weak force converts an electron into a linear superposition of neutrinos from different generations.

Since it's not possible to create linear superpositions of electrons and neutrinos, it is only possible to make statistical mixtures of these particles. This is the same case as the classical beams we considered in the previous section. Classical beams are represented by matrices of particle rates that are diagonal as in Equation (6). Before we split the particle rates into bras and kets, we represented spin-1/2 particles with $2 \times 2$ Hermitian matrices.

By reconsidering our splitting we can create a $4 \times 4$ matrix that represents a statistical mixture of electrons and neutrinos, each of which is a quantum superposition of spin-up and spin-down. Suppose our beam is $40 \%$ electrons with spin-1/2 in the $+u$ direction and $60 \%$ neutrinos with spin-1/2 in the $+V$ direction. Then the matrix representation is:

$$
\begin{aligned}
\rho(T) & =0.4|+u, e\rangle\langle+u, e|+0.6|+v, v\rangle\langle+v, v|=0.4 \rho_{+u, e}+0.6 \rho_{+v, v} \\
& =\left(\begin{array}{cccc}
0.2+0.2 u_{z} & 0.2 u_{x}-0.2 i u_{y} \\
0.2 u_{x}+0.2 i u_{y} & 0.2-0.2 u_{z} & & \\
& & 0.3+0.3 v_{z} & 0.3 v_{x}-0.3 i v_{y} \\
& 0.3 v_{x}+0.3 i v_{y} & 0.3-0.3 v_{z}
\end{array}\right)
\end{aligned}
$$


where $T$ is some arbitrary temperature and, for the sake of clarity, we've left the forbidden matrix entries blank instead of zero. This matrix is in the form of a mixed density matrix subject to the requirement that its bras and kets not cross the superselection sector boundary. That is, the kets are split into two halves and only one of the halves can be non zero. If it is the top half, then the ket is an electron ket while the bottom half defines a neutrino ket.

We've just shown that the particle rate matrix for beams that include more than one superselection sector must be in block diagonal form. The splitting of two superselection sectors is a classical beam splitter and this can always be done. After reducing a beam to a single superselection sector we can do quantum measurements on it with the methods discussed above. We can do this to both the particle types and then reassemble the two beams into one. This way we can design a beam splitter whose output stream will have the properties of $40 \%$ electron and $60 \%$ neutrino beam discussed above, at least given an input stream with both electrons and neutrinos.

We can also consider thermal measurements of beams composed of particles from two different superselection sectors. As before with Equation (42), the high temperature limit for each superselection sector will be a multiple of the unit matrix for that sector. If we balance the sectors to have the same multiple, the high temperature limit will be a multiple of unity. Setting the trace to be 1, the high temperature limit for the electron/neutrino block diagonal matrix will be:

$$
\frac{1}{4}\left(\begin{array}{cccc}
1 & 0 & & \\
0 & 1 & & \\
& & 1 & 0 \\
& & 0 & 1
\end{array}\right),
$$

where again we've left the forbidden entries blank rather than zero.

We can reduce the temperature of the above matrix $\rho(T)$ in Equation (46) by squaring and renormalizing to keep the trace 1 . This is easily done in the form $0.4 \rho_{+u, e}+0.6 \rho_{+v, v}$ as $\rho_{+u, e}$ and $\rho_{+v, v}$ are idempotent, annihilate each other and have unit trace. We find:

$$
\rho(T)^{n} / \operatorname{tr}\left(\rho(T)^{n}\right)=\left((0.4)^{n} \rho_{+u, e}+(0.6)^{n} \rho_{+v, v}\right) /\left((0.4)^{n}+(0.6)^{n}\right)
$$

In the limit as $n \rightarrow \infty$ the $(0.6)^{n}$ terms dominate giving a limit

$$
\rho(0)=\rho_{+v, v} .
$$

It should be clear that this is a general attribute of quantum beam splitters that cross superselection sectors. That is, their $T=0$ limit falls into a single sector.

If we are able to make calculations in an algebra and want to know what particles it contains this implies an algorithm. We begin with the high temperature limit of Equation (47) and add a small Hermitian modification to it. Then repeatedly square and renormalize the trace to unity. It will approach the primitive idempotent for a particle. 
For our example of the electron/neutrino, the operator for electric charge is zero in the neutrino part and $-\hat{1}=-1 \hat{1}$ in the electron part:

$$
Q=\left(\begin{array}{cccc}
-1 & 0 & & \\
0 & -1 & & \\
& & 0 & 0 \\
& & 0 & 0
\end{array}\right)
$$

This matrix commutes with any element of the measurement algebra for the electron/neutrino beam. In fact, the definition of a charge that creates superselection sectors is that the symmetry must commute with every possible measurement (observable).

Given an algebra, we can define the possible charges that define its superselection sectors. Each diagonal block can take a different charge. For the electron/neutrino algebra, the possible charges are:

$$
Q\left(q_{e}, q_{v}\right)=\left(\begin{array}{cccc}
q_{e} & 0 & & \\
0 & q_{e} & & \\
& & q_{v} & 0 \\
& & 0 & q_{v}
\end{array}\right) .
$$

where $q_{e}$ and $q_{v}$ are the charges for the electron and neutrino blocks. This observation is trivial for block diagonal algebras but becomes interesting when the algebra is defined more subtly.

\section{Standard Model/Dark Matter}

The Standard Model of elementary particles is built around symmetries. This is a natural consequence of the experiments; humans look for patterns in the experimental results and the nicest way to define patterns is with symmetry. So the quantum mechanics that models the elementary particles is defined using the symmetries that are observed in the experiments. While this method is clearly the easiest way of obtaining a model that matches experiment, it should also be clear that the seeking of symmetries is a human attribute and not necessarily an indication of how Nature is most easily understood. Here we explore an alternative way of describing elementary particles.

The Standard Model of elementary particles consists of representatives of $\mathrm{SU}(3) \times \mathrm{SU}(2) \times \mathrm{U}(1)$ symmetry. Why did nature choose this symmetry rather than, for example, $\mathrm{SU}(4) \times \mathrm{E} 4 \times \mathrm{U}(1) \times \mathrm{U}(1)$ ? The Standard Model provides no explanation for the arbitrariness of the choice of symmetry. On the other hand, what is interesting here is that $\mathrm{SU}(3) \times \mathrm{SU}(2) \times \mathrm{U}(1)$ is a block diagonal symmetry.

It is not enough to choose the symmetry $\mathrm{SU}(3) \times \mathrm{SU}(2) \times \mathrm{U}(1)$. The particles are taken as irreducible representations of this symmetry so one must also choose these irreps. To define an irrep of $\mathrm{SU}(3) \times \mathrm{SU}(2) \times \mathrm{U}(1)$ one chooses an irrep of $\mathrm{SU}(3)$, one of $\mathrm{SU}(2)$ and one of $U(1)$. Each of these has an infinite number of choices; the ones used by the fermions in the Standard Model are: 


\begin{tabular}{ccc|c}
$\mathrm{SU}(3)$ & $\mathrm{SU}(2)$ & $\mathrm{U}(1)$ & Particles \\
\hline 1 & 1 & -2 & right-handed electron \\
1 & 1 & 0 & right-handed neutrino \\
1 & 2 & -1 & left-handed leptons \\
3 & 1 & $4 / 3$ & right-handed upquark \\
3 & 1 & $-2 / 3$ & right-handed downquark \\
3 & 2 & $1 / 3$ & left handed quarks
\end{tabular}

Why did Nature choose these six representations? And why do they appear in three generations identical except for mass and the weak force? The Standard Model provides no explanations for the arbitrariness of the above choice of symmetry representations.

What we would like is a single mathematical object that is natural, and reproduces the arbitrary choices of the Standard Model. To do that, we can assume that the symmetry and representations both arise from a single choice of algebra instead of a choice of symmetry. One problem with this idea is that $\mathrm{SU}(3) \times \mathrm{SU}(2) \times \mathrm{U}(1)$ is a gauge symmetry and the tool this paper develops is the particle content. In addition, the SU(2) portion of the Standard Model symmetry is unbroken only in the high temperature limit. This paper looks at putting several particles into a single density matrix due to the consequences of block diagonal form. Another way of putting multiple representations into mixed density matrices is explored in [8].

At normal temperatures, the weak SU(2) symmetry that relates the left handed electron and neutrino is broken. In fact, left handed fermions travel at speed $c$ and so do not appear at all at zero temperature. This implies that the zero temperature particle content needs to deal with the electron, neutrino, up-quark and down-quark rather than their left and right handed components. Under this assumption, there are 4 Standard Model fermions. Ignoring spin and anti particles, the electron and neutrino will appear as two $1 \times 1$ blocks. The up and down quarks are color $\mathrm{SU}(3)$ triplet states so they will appear as two $3 \times 3$ blocks.

If we have a finite group $G$, we can create an algebra from it that is called $\mathbb{C}(G)$, the "complex group algebra". A complex group algebra can be put into block diagonal form [12]. We can then read the particle content off by noting the size of the blocks. Again, note that the particle content is not the same as a gauge symmetry.

In Subsection 3.2 we showed that consecutive measurements in the $+z,+x,+y$ and $+z$ directions resulted in the beam picking up a geometric phase of $-\pi / 4$. On the other hand, any complex plane wave picks up a phase through translation in space or time according to $\exp (i k x-\omega t)$. We cannot distinguish these two effects; either one will give a phase change to a beam that we can detect by seeing changes in an interference pattern. If Nature were truly simple, these two causes of phase changes would be the same. For that to happen, the movement of spin-1/2 particles would have to be accompanied by changes in their spin direction [13]. 
The left and right handed fermions of Equation (52) are massless and travel at speed $c$. A stationary electron with spin- $1 / 2$ in the $+z$ direction is produced by combining a right handed electron with speed $c$ in the $+z$ direction and a left handed electron with speed $c$ in the $-z$ direction. The "Feynman checkerboard model" of the electron is a 1-dimensional model of the Dirac equation based on sums over discrete paths on the corners of a checkerboard [14]. The axes on the checkerboard correspond to position $z$ and time $t$. Each step in a path the electron moves one unit forward in time by $\epsilon$ and steps by a distance $\pm \epsilon C$ in $z$. So the electron is moving up the checkerboard on diagonal lines, the way a checker moves in a game of checkers or draughts. Each time the electron changes direction the path receives a factor of $-i \epsilon m c^{2} / \hbar$. In the $\epsilon \rightarrow 0$ limit, the sum of all paths gives a propagator that satisfies the 1-d Dirac equation.

The 1-dimensional Feynman checkerboard paths have been extended to 3-dimensional [15] [16]. The particle paths in space are on the points of a cubic lattice. The possible symmetries of a cubic lattice are defined by the isometric crystallographic point groups:

$$
\begin{array}{c|ccccc}
\text { Group: } & T & T_{h} & O & T_{d} & O_{h} \\
\text { Size: } & 12 & 24 & 24 & 24 & 48
\end{array}
$$

in Schoenflies notation.

The block diagonal structure of a complex group algebra can be read off of the group character table. Each irreducible character corresponds to a block on the diagonal and the size of the block is given by the irreducible character of the identity $E$ [12]. For example, the character table for $T$ is given by

\begin{tabular}{c|cccc}
$T$ & $E$ & $C_{2}$ & $C_{3}$ & $C_{3}^{\prime}$ \\
Size: & 1 & 3 & 4 & 4 \\
\hline$A$ & 1 & 1 & 1 & 1 \\
$E$ & 1 & 1 & $\omega$ & $\omega^{2}$ \\
$E^{\prime}$ & 1 & 1 & $\omega^{2}$ & $\omega$ \\
$T$ & 3 & -1 & 0 & 0
\end{tabular}

where $\omega=\exp (2 i \pi / 3)$ and "Size" is the number of group elements in that class. For a derivation of this character table see Section 4-1, table 4-6 in [12]. Each row corresponds to an irreducible representation of the symmetry. There are four: $A, E, E^{\prime}, T$ and each irrep will appear in the algebra as a block on the diagonal. So there are four blocks in $\mathbb{C}(T)$; three of size $1 \times 1$ and one of size $3 \times 3$.

A general element of the $\mathbb{C}(T)$ algebra, in block diagonal form, looks like:

$$
\left(\begin{array}{cccccc}
\alpha & & & & \\
& \eta & & & & \\
& & \eta^{\prime} & & & \\
& & \tau_{11} & \tau_{12} & \tau_{13} \\
& & \tau_{21} & \tau_{22} & \tau_{23} \\
& & & \tau_{31} & \tau_{32} & \tau_{33}
\end{array}\right)
$$


where $\alpha, \eta, \eta^{\prime}$ and $\tau_{j k}$ are complex numbers. For the Standard Model fermions we need two $1 \times 1$ blocks for the leptons and two $3 \times 3$ blocks for the quarks so $T$ symmetry will not suffice. As we can count from Equation (55), the total number of complex degrees of freedom in the $\mathbb{C}(T)$ algebra is $1^{2}+1^{2}+1^{2}+3^{2}=12$. This is the same as the size of the finite group $T$. In other words, putting the algebra into block diagonal form is just a way of rewriting its degrees of freedom.

Examining the character tables of the five isometric crystallographic point groups [12] we find that their particle content consists of:

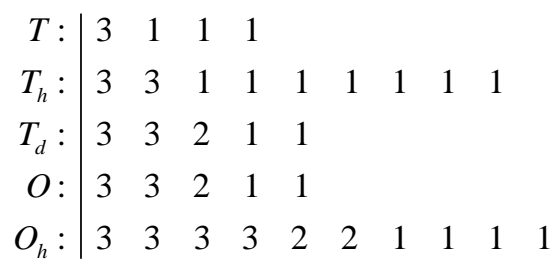

and we find that all but the $T$ group include the 3311 particle content of the Standard Model. Of these five groups, $T_{h}$ and $O_{h}$ include an inversion $i$ or point reflection. Such a symmetry transforms an object into its mirror image. In the Standard Model the weak interaction is not symmetric under parity so we will reject those groups.

This leaves $T_{d}$ and $O$ which share the same characters:

\begin{tabular}{r|rrr|rr}
$O:$ & $E$ & $C_{3}$ & $C_{2}$ & $C_{2}^{\prime}$ & $C_{4}$ \\
$T_{d}:$ & $E$ & $C_{3}$ & $C_{2}$ & $\sigma_{d}$ & $S_{4}$ \\
Size: & 1 & 8 & 3 & 6 & 6 \\
\hline$A_{1}:$ & 1 & 1 & 1 & 1 & 1 \\
$A_{2}:$ & 1 & 1 & 1 & -1 & -1 \\
$E:$ & 2 & -1 & 2 & 0 & 0 \\
$T_{1}:$ & 3 & 0 & -1 & -1 & 1 \\
$T_{2}:$ & 3 & 0 & -1 & 1 & -1
\end{tabular}.

In the above table, we've included an extra vertical line between the first three conjugacy classes and the last two. This is to note that $E, C_{3}$ and $C_{2}$ are even while the two right classes are odd. Even and odd are determined by the $A_{2}$ or "sign" irrep. We assign the $A_{j}$ pair to the leptons and the $T_{j}$ pair to the quarks. These pairs differ only in their odd characters so we presume that it is the odd characters that define their weak isospin and weak hypercharge quantum numbers of the handed Standard Model fermions. The remaining irrep $E$ is distinctive in that it has no odd characters. So we assume that it corresponds to a particle with no weak hypercharge or weak isospin and assign it to dark matter.

\section{Conclusions}

We've shown that binary measurements are quite similar whether they are classical or quantum. Since binary measurements form a foundation for 
quantum mechanics as demonstrated in the Schwinger measurement algebra textbook introductions to quantum mechanics [5] [6], this shows that this formulation of quantum mechanics is more closely aligned with our classical intuition than the other formulations. This makes this formulation important for pedagogy as well as the foundations of quantum mechanics.

The paper shows a new way of introducing quantum mechanics to students from the point of view of classical mechanics. This allows students to have an intuitive understanding of how quantum mechanics works. We've shown how the use of complex numbers and amplitudes instead of probabilities is natural to quantum mechanics.

Some of the subjects discussed in this paper including quantum phase, Berry-Pancharatnam phase, superselection sectors and quantum statistics are introduced in this paper at an elementary level. These subjects provide interesting subjects to study for students in their first lectures on quantum mechanics with interesting problems available for assignment.

Finally, we've illustrated the possible uses of this new formulation of classical and quantum mechanics in explorations of the nature of the elementary fermions. We show that the choice of an algebra defines both symmetry and particle representations. This may be a method of wrapping the symmetries of the Standard Model into an algebraic description that will be much more tightly determined than the arbitrary choice of symmetry and representations found in the Standard Model.

\section{Acknowledgements}

Thanks to the faculty at Washington State University for their encouragement and assistance, to Mark Mollo and Joseph. P. Brannen for financial support, and to the grad students at WSU for their fellowship, and the anonymous reviewers for improvements to the text.

\section{References}

[1] Schwinger, J. (1959) Proceedings of the National Academy of Science, 45, 1542-1553. http://www.pnas.org/content/45/10/1542.full.pdf

[2] Schwinger, J. (1960) Proceedings of the National Academy of Science, 46, 257-265. http://www.pnas.org/content/46/2/257.full.pdf

[3] Schwinger, J. (1960) Proceedings of the National Academy of Science, 46, 883-897. http://www.pnas.org/content/46/6/883.full.pdf

[4] Schwinger, J. (1960) Proceedings of the National Academy of Science, 46, 1401-1415. http://www.pnas.org/content/46/10/1401.full.pdf

[5] Schwinger, J. and Englert, B. (2001) Quantum Mechanics: Symbolism of Atomic Measurements. Springer, Berlin. https://doi.org/10.1007/978-3-662-04589-3

[6] Schwinger, J. (2000) Quantum Kinematics and Dynamic. Advanced Books Classics, Avalon Publishing, New York.

[7] Styer, D.F., Balkin, M.S., Becker, K.M., Burns, M.R., Dudley, C.E., Forth, S.T., Gaumer, J.S., Kramer, M.A., Oertel, D.C., Park, L.H., Rinkoski, M.T., Smith, C.T. and 
Wotherspoon, T.D. (2002) American Journal of Physics, 70, 288-297.

https://doi.org/10.1119/1.1445404

[8] Weinberg, S. (2014) Physical Review A, 90, Article ID: 042102.

https://doi.org/10.1103/PhysRevA.90.042102

[9] Ellerman, D. (2011) A Very Common Fallacy in Quantum Mechanics: Superposition, Delayed Choice, Quantum Erasers, Retrocausality, and All That.

[10] Storer, R.G. (1968) Journal of Mathematical Physics, 9, 964-970.

https://doi.org/10.1063/1.1664666

[11] Giulini, D., Kiefer, C. and Zeh, H.D. (1995) Physics Letters A, 199, 291-298. https://doi.org/10.1016/0375-9601(95)00128-P

[12] Hamermesh, M. (1962) Group Theory and Its Application to Physical Problems. Dover Publications, Mineola.

[13] Brannen, C.A. (2010) Foundations of Physics, 40, 1681-1699. https://doi.org/10.1007/s10701-010-9465-8

[14] Feynman, R.P. and Hibbs, A.R. (1965) Quantum Mechanics and Path Integrals. McGraw-Hill, New York.

[15] Jacobson, T. (1985) Feynman's Checkerboard and Other Games. Springer, Berlin Heidelberg, 386-395.

[16] Smith, F.D. (1995) Hyperdiamond Feynman Checkerboard in Four-Dimensional Space-Time. 